\title{
Über die Autorinnen und Autoren
}

Dr. iur. Wolfgang Besler war zehn Jahre Richter am Verwaltungsgericht, sodann Beigeordneter der Stadt Iserlohn. Seit 2005/2006 ist er niedergelassener Rechtsanwalt und Lehrbeauftragter für das Fach Schulrecht am Institut für Öffentliches Recht und Politik (Prof. Dr. Bodo Pieroth/Prof. Dr. Fabian Wittreck) der Westfälischen Wilhelms-Universität Münster.

Dr. phil. Dirk Hänisch ist Diplomsozialwissenschaftler und war am Zentralinstitut für sozialwissenschaftliche Forschung der Freien Universität Berlin tätig. Zahlreiche Veröffentlichunen zur historischen Wahlforschung (dirkhaenisch@yahoo.de).

Anja Heidelberger arbeitet als Assistentin am Institut für Politikwissenschaft der Universität Bern (anja.heidelberger@ipw.unibe.ch).

Prof. Dr. iur. Hermann K. Heußner lehrt Öffentliches Recht und Recht der sozialen Arbeit an der Fachhochschule Osnabrück (heussner@wi.fh-osnabrueck.de).

Benjamin Karras ist Wissenschaftlicher Mitarbeiter am Institut für Öffentliches Recht und Politik der Westfälischen Wilhelms-Universität Münster.

Stefan Lenz ist studentische Hilfskraft am Kommunalwissenschaftlichen Institut der Westfälischen Wilhelms-Universität Münster.

Miriam Minder, MLaw, arbeitet als Assistentin am Institut für Öffentliches Recht der Universität Bern (miriam.minder@oefre.unibern.ch).

Dr. iur. Maria Pottmeyer, LL. M. (Cambridge) war Akademische Rätin a.Z. am Kommunalwissenschaftlichen Institut der Westfälischen Wilhelms-Universität Münster und Leiterin des dortigen Freiherr-vom-Stein-Instituts.

Univ.-Prof. Dr. iur. Dian Schefold lehrte Öffentliches Recht, einschl. allgemeine Staatslehre und neuere Verfassungsgeschichte mit dem Schwerpunkt Verwaltungsrecht an der Universität Bremen.

Univ.-Prof. Dr. iur Axel Tschentscher, LL. M. (Cornell) lehrt Öffentliches Recht, Rechtsphilosophie und Verfassungsgeschichte an der Universität Bern (axel.tschentscher@oefre. unibe.ch).

Univ.-Prof. Dr. rer. pol. Adrian Vatter ist Direktor des Instituts für Politikwissenschaft an der Universität Bern (adrian.vatter@ipw.unibe.ch).

Univ.-Prof. Dr. iur. Ferdinand Wollenschläger ist Inhaber des Lehrstuhls für Öffentliches Recht, Europarecht und Öffentliches Wirtschaftsrecht an der Juristischen Fakultät der Universität Augsburg. 